\title{
Variable Data Flow Management in Wireless Sensing Network for Landslide Disaster
}

\author{
S. Takayama, M. Hiraoka, K. Mori, K. Kariya \\ D.E.E.Engng., Faculty of Science and Engineering, Ritsumeikan Univ. BKC \\ Noji, Kusatsu, Shiga, 525-8577, JAPAN \\ Email: s-tkym@se.ritsumei.ac.jp
}

\begin{abstract}
Sensing systems to monitor natural disasters face many hard conditions. Natural disasters occur suddenly, and damage the sensor system. Therefore, the sensor system should be designed as a distributed node network. In addition to that, the network should have some characteristic functions, such as self-recovery, autonomous operation and effective data transmission. This paper describes an effective data flow management in wireless sensing node network for landslide disasters. The sensing node network is operated by three modes (initializing, measuring and urgent). By switching these operation modes autonomously, the sensing node network becomes a robust system (loss/insert) of sensing node and the dynamic control of data transmission. Finally, the effectiveness of the operation is supported by some experiments.
\end{abstract}

Keywords: sensing network, landslide monitoring

\section{INTRODUCTION}

$\mathbf{I}_{\mathrm{p}}^{\mathrm{t}}$ is extremely difficult to predict exactly the time, date and place of occurrence of natural disasters such as landslides.

Therefore, diary monitoring around the dangerous area is important. If we can get indications before the occurrence, the information is very helpful for reporting to inhabitants and for the operation of the monitoring system[1-3]. To monitor landslide disasters, the monitoring system should have some characteristic functions. The monitoring system should transmit precision measuring data urgently to the host system corresponding to the disaster occurrence. As the occurrence place of a disaster is not known exactly, it is necessary to monitor an area by using distributed sensing nodes. The system should prevent the loss of measuring data (by redundant data storage) with cooperation among the sensing nodes. As the sensing node is easily damaged and thus its functions are stopped, the sensing node network should have a self-recovery function.

This paper describes the construction of a sensing node network system which operates flexibly towards disappear/insert of sensing nodes and urgent data transmission. The operation is shown by some examples of landslide disaster monitoring .

\section{LOCAL SENSING NODE NETWORK FOR LANDSLIDE DISASTER}

Fig. 1 shows an example of a local network of sensing nodes. The local network is connected to the host system by long distance communication function of Node0. The sensing node provides for the following functions: measuring, data processing, temporary memory and communication. To monitor a landslide, plural sensing nodes are distributed on a closed area.
These sensing nodes form an autonomously constructed tree-type local network. Node0 is a special node to communicate a message (commands and measuring data) to the host system. Then the Node0 has wireless communication unit (out of network) with normal wireless communication function among sensing nodes. Each sensing node regularly measures land movement, acceleration of landslide and temperature. Usually, stationary measuring data is stored temporarily. Simultaneously, they are also stored by the surrounding sensing nodes redundantly.

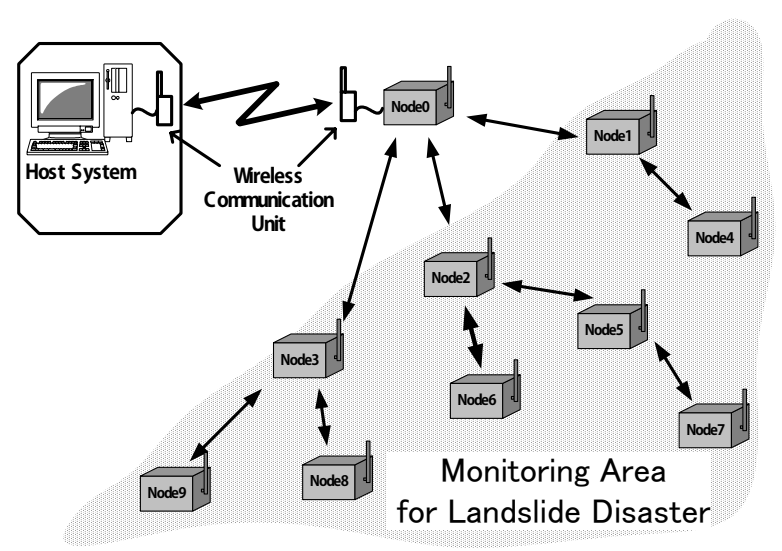

Fig.1 Example of local sensing nodes network

The local sensing node network has three characteristic operation modes (initialing, measuring, urgent ).

The initializing mode is the operation mode for an autonomous construction of a network of sensing nodes. 
The mode plays important roles in

1) the first construction of a local network,

2) urgent transmission of the measuring data to the host system,

3) the loss of sensing nodes by a landslide disaster.

In the measuring mode, for the monitoring of the indication of occurrence of a landslide, the sensing node measures a signal and stores the commonly shared measuring data in the surrounding sensing nodes. To enhance the communication efficiency in the local network, the sensing node measures by irregular sampling time interval based on stochastic parameter. In case of a small difference in fluctuations of measuring data, the sensing node measures by a long sampling interval. On the other hand, in case of a large difference in fluctuations of measuring data or if a threshold level is exceeded, the sensing node measures by a short sampling interval. Additionally, the measuring mode inserts a new sensing node in the local network to expand the monitoring area and to supplement the missing sensing node.

The urgent mode is the mode for urgent transmission of measuring data in the local network when a sensing node discovers an indication of a landslide disaster and senses its occurrence. The urgent mode resets the tree-type network construction temporarily and realizes a strait connection between Node0 and the sensing node through other nodes.

\section{DATA FLOWS IN A SENSING NODE NETWORK}

\section{Frequency of measuring data in Node}

To observe the operation of the sensing node by three operation modes, the data flow in the sensing node is monitored. The local network is constructed of five sensing nodes (Node0, Node1, Node2, Node3, Node4). Node0 is for the communication with the host system. Node1 mounts a soil moisture sensor. Node2 and Node4 mount acceleration sensors. Node3 mounts a temperature sensor. The local sensing node network was tested by the following timing diagram.

1) First period : 0-60 min.

*Initializing mode after power- on of all nodes

The first measuring mode (Operation nodes are Node0, 1, 2, 3)

2) Second period : $60-120 \mathrm{~min}$.

*Initializing mode after a shock to acceleration sensor in Node2

*Urgent mode by Node2 (Operation nodes are Node0, 2)

3) Third period : 120-180min.

*Initializing mode after power off of Node2

[instead of loss of Node2]

*The second measuring mode after loss of Node2

(Operation nodes are Node0, 1, 3)

4) Fourth period : 180-210min.

*Initializing mode after insert of Node4

*The third measuring mode after insert of Node4

(Operation nodes are Node0, 1, 3, 4)

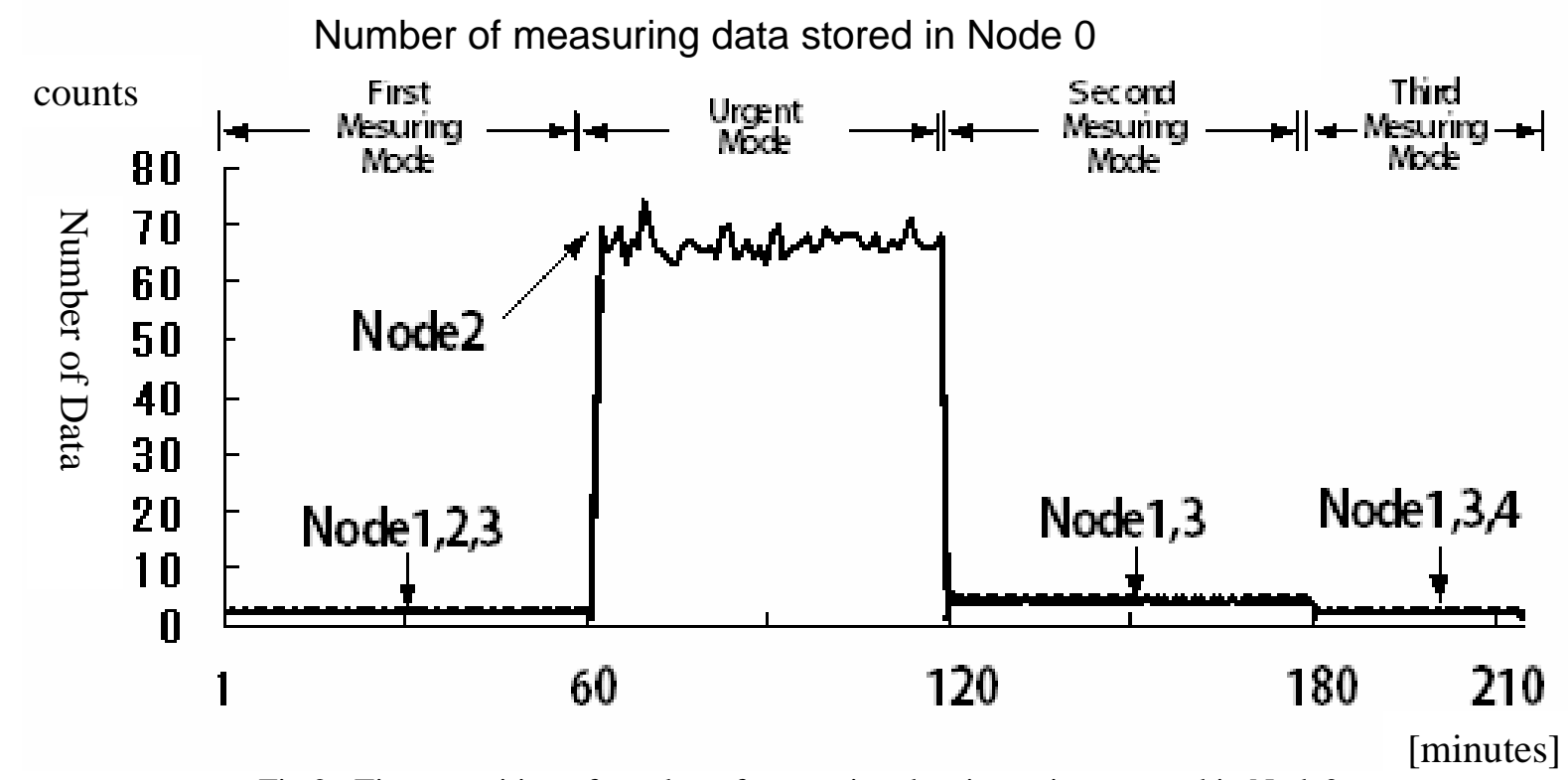

Fig.2 Time transition of number of measuring data in a minute stored in Node0 
Fig.2 shows the time transition of the number of measuring data in a minute stored in Node0. The time for initializing modes reconstructing the local network is within one minute. In the first measuring mode, the number of measuring data of Node1,2,3 is stored in the same stationary. In the urgent mode by Node2, sampling time interval of Node2 becomes short. The frequency of data transmitted to Node0 becomes high. As a result, the storage in Node 0 is only measuring the data of Node2. In the second measuring mode, as the number of sensing nodes becomes lower, the number of measuring data increases, if compared with the first measuring mode. As the number of sensing nodes in the third measuring mode becomes the same as in the first measuring mode, then, the number of measuring data is the same.

\section{Observation of measuring data}

To confirm the measuring data controlled by three operation modes, the data are monitored by the host system. The local network is constructed of five sensing nodes (Node0, Node2, Node3, Node5, Node6). Node2 mounts a temperature sensor. Node3 mounts a torque sensor. Node5 mounts a soil moisture sensor. Node6 mounts an acceleration sensor. Fig.3 presents the node network for test operation. The local sensing node network was tested by the following timing diagram.

1) First period : 0-15 min.

*Initializing mode after power on of all nodes

*The first measuring mode

(Operation nodes are Node $0,2,3,5,6$ )

2) Second period : $15-25 \mathrm{~min}$.

*Initializing mode after an urgent response of

soil moisture sensor in Node5

*The first urgent mode by Node5

(Operation nodes are Node0, 5)

3) Third period : 25-40min.

*Initializing mode after power off of Node5

[instead of loss of Node5]

*The second measuring mode after loss of Node5 (Operation nodes are Node0, 2, 3, 6)

4) Fourth period : 40-45min.

*Initializing mode after a shock to acceleration sensor in Node6

*The second urgent mode by Node6

(Operation nodes are Node0, 6)

5) Fifth period : 45-60min.

*Initializing mode after power off of Node6

6) Sixth period : 60-75min.

*The third measuring mode after loss of Node6 (Operation nodes are Node0, 2, 3)

[instead of loss of Node6]
*Initializing mode after insert of Node5

*The fourth measuring mode after insert of Node5

(Operation nodes are Node0, 2, 3, 5)

7) Seventh period : 75-90min.

*Initializing mode after insert of Node6

*The fifth measuring mode after insert of Node6

(Operation nodes are Node0, 2, 3, 5, 6)

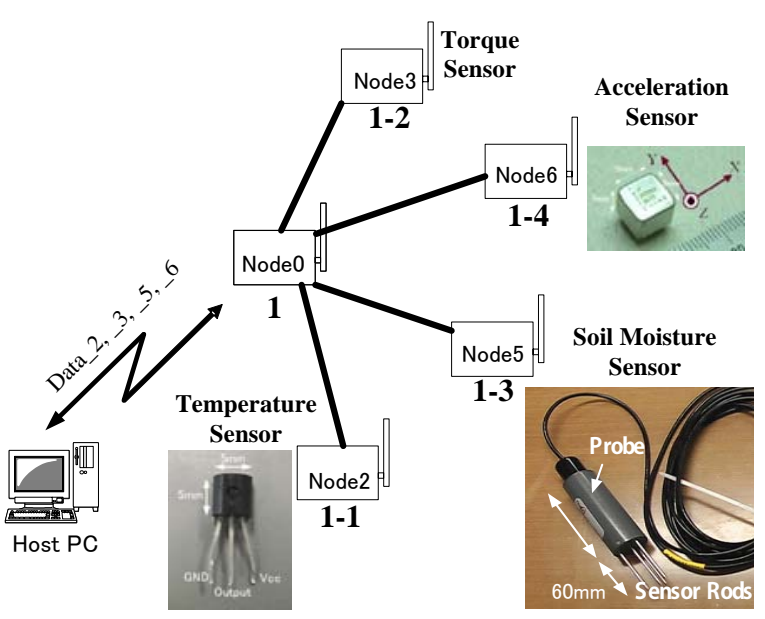

Fig.3 Simple node network to monitor measuring data

Fig. 4 shows the time transition of the number of measuring data in a minute stored in the host system.

In the first measuring mode, the number of measuring data of Node2,3,5,6 is stored in the same stationary. In the first urgent mode caused by Node5, the measuring data (soil moisture) of Node5 are only transmitted. Other data are stopped. Fig. 5 shows the time transition of measuring data of soil moisture (Node5) stored in the host system. It is confirmed that the frequency of measuring data of soil moisture is high. In the second measuring mode, the number of measuring data of Node2,3,6 is stored in the same stationary. As Node5 was lost, the data are not monitored. In the second urgent mode caused by Node6, the measuring data (acceleration) of Node6 are only transmitted. Fig. 6 shows the time transition of measuring data of acceleration (Node6) stored in the host system. It is confirmed that the frequency of measuring data of acceleration is high. In the third measuring mode, the number of measuring data of Node2,3 is stored in the same stationary. As Nodes5,6 were lost, the data are not monitored. In the fourth measuring mode, Node5 is restarted. 


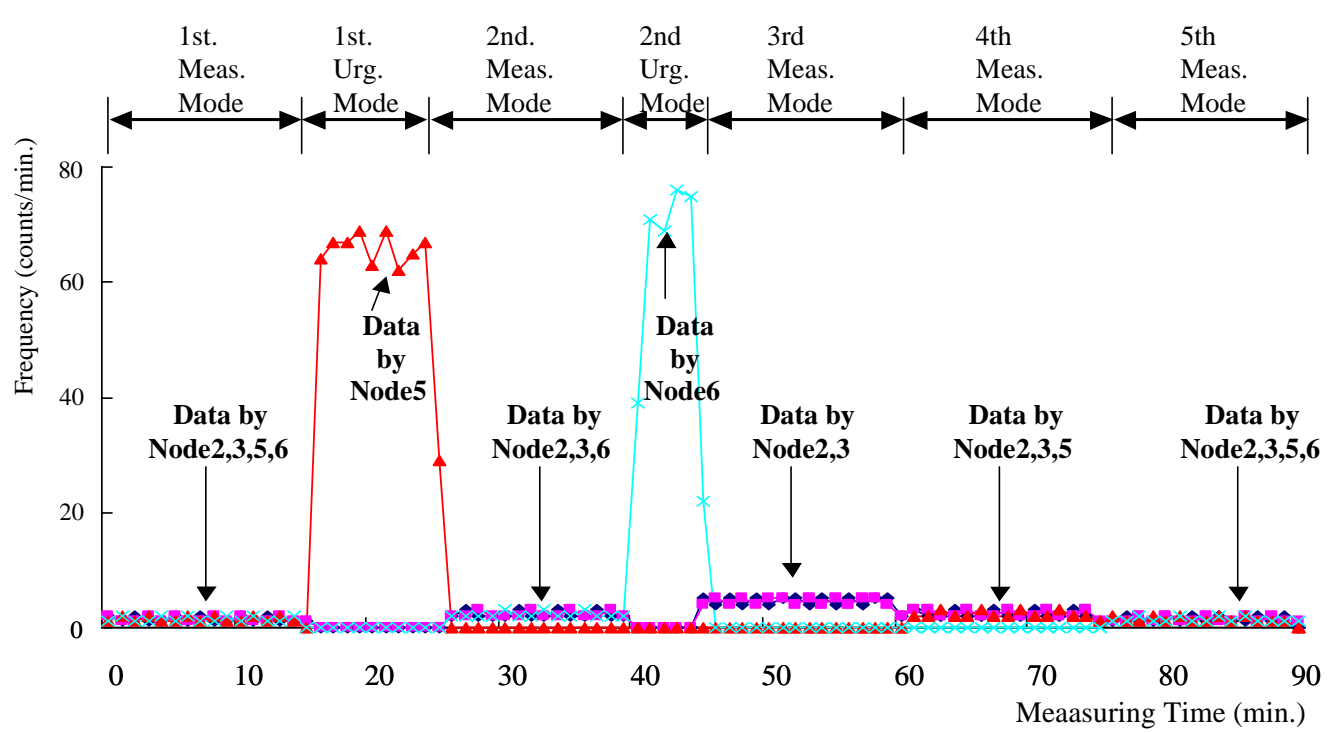

Fig.4 Time transition of number of measuring data in a minute stored in host system

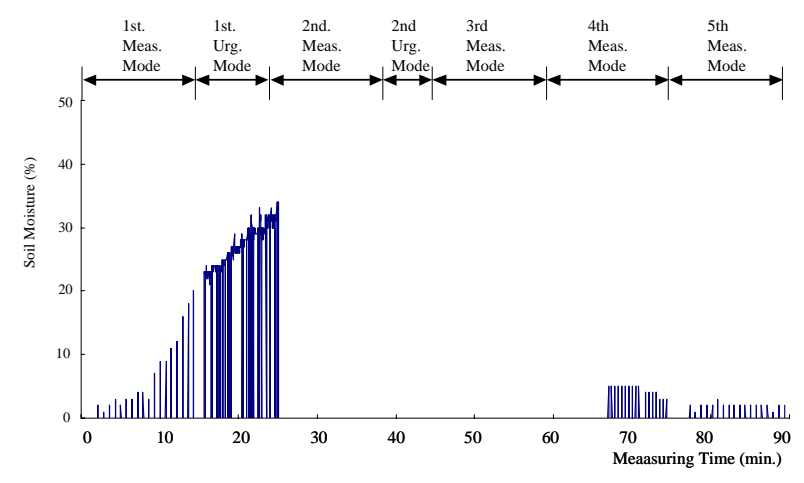

Fig.5 Time transition of measuring data of soil moisture in Node5

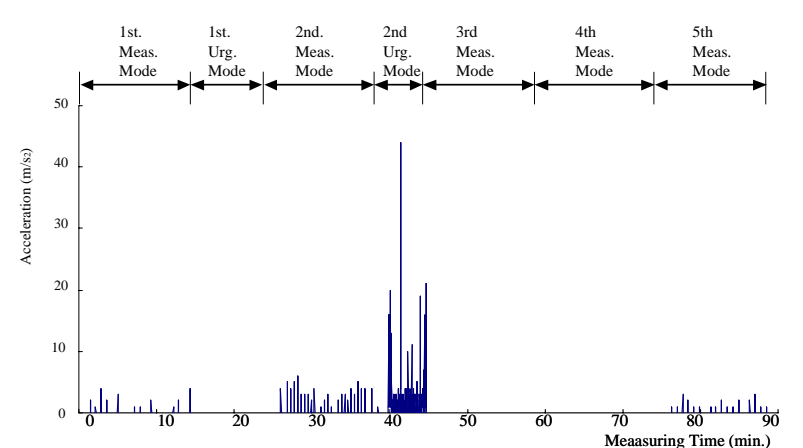

Fig.6 Time transition of measuring data of acceleration sensor in Node6

\section{CONCLUSIONS}

The time of 10-20 minutes before the occurrence of a disaster can save many human lives. To monitor landslide disasters, attention must be paid to ensure that a sensor node transmits the data continuously at a sign or occurrence of a landslide. Further, it should be noted that a sensor node can be broken easily by a landslide disaster. Therefore, it is necessary to construct a robust and flexible sensing system. In this paper, an autonomous monitoring system using local sensing node network is proposed. We have shown that the sensing node network has some characteristic functions, including node self-recovery, flexible node arrangement, and effective data transmission during a disaster.

\section{REFERENCES}

[1] Information Communication System of Disasters Prevention Association (2003). Information Communication System of Disasters Prevention (pp. 173), Sankaido.

[2] Kyoto University Disasters Prevention Laboratory (2003). Foundation Disaster (pp. 9-21). Sankaido,

[3] Takei, A. (1980). Landslide, collapse, mudslide Prediction and Measure (pp.65-77). Kashima publishing. 